\title{
Bone bruises in anterior cruciate ligament injured knee and long-term outcomes. A review of the evidence
}

This article was published in the following Dove Press journal:

Open Access Journal of Sports Medicine

18 February 2015

Number of times this article has been viewed

\author{
Rocco Papalia' \\ Guglielmo Torre' \\ Sebastiano Vasta' \\ Biagio Zampogna' \\ Douglas R Pedersen ${ }^{2,3}$ \\ Vincenzo Denaro' \\ Annunziato Amendola ${ }^{3}$ \\ 'Department of Orthopaedic and \\ Trauma Surgery, Campus Bio- \\ Medico University of Rome, Rome, \\ Italy; ${ }^{2}$ Department of Biomedical \\ Engineering, ${ }^{3}$ Department of \\ Orthopaedics and Rehabilitation, \\ University of lowa, lowa City, IA, USA
}

Background: Bone bruises are frequently associated with anterior cruciate ligament (ACL) tears as a result of trauma or direct shear stress of the bone.

Purpose: To review the evidence regarding the characteristics of the bone bruise associated with ACL tears, its relevance on clinical outcomes, and its progression over time. In particular, the long-term effects of the bone bruise on the knee osteochondral architecture and joint function were evaluated.

Study design: Review; level of evidence: 4.

Methods: An electronic search was performed on PubMed. Combinations of keywords included: "bone bruise AND knee"; "bone bruise AND anterior cruciate ligament"; "bone bruise AND osteo-chondral defects". Any level of evidence studies concerning bone bruises in patients with partial or complete ACL tears were retrieved.

Results: A total of 25 studies were included; three of them investigated biomechanical parameters, seven were concerned with clinical outcomes, and 15 were radiological studies. Evaluation of the bone bruise is best performed using a fat-saturated T2-weighted fast spin echo exam or a short tau inversion recovery sequence where fat saturation is challenging. The location of the injury has been demonstrated to be more frequent in the lateral compartment of the joint (lateral femoral condyle and lateral tibial plateau). It is associated with ACL tears in approximately $70 \%$ of cases, often with collateral ligament or meniscal tears. Mid- and longterm outcomes demonstrated a complete healing of the marrow lesions at magnetic resonance imaging, but chondral defects detected with T1 $\rho$ sequences are still present 1 year after the ACL injury. Functional examination of the knee, through clinical International Knee Documentation Committee scores, did not show any correlation with the bone bruise

Conclusion: Although bone bruise presence yields to higher pain levels, no correlation with functional outcomes was reported. Most studies have a short-term follow-up ( $<2$ years) compared to the length of time it takes to develop post-traumatic osteoarthritis, so it still remains unclear whether the initial joint injury and bone bruise have a direct relationship to long-term function.

Keywords: bone bruise, anterior cruciate ligament, magnetic resonance imaging, knee

\section{Introduction}

Anterior cruciate ligament (ACL) tears are commonly caused during sport participation $^{1}$ and are often associated with meniscal and cartilage lesions. The global incidence of ACL injuries is about 100,000-250,000 cases per year. ${ }^{2}$ A high rate (from 10\%-90\%) of osteoarthritis development after ACL injury has been reported, despite optimizing treatment, ${ }^{3-6}$ in mid- and long-term follow-up studies (up to 14 years). Magnetic resonance imaging (MRI) is currently the best imaging tool for radiological
Correspondence: Annunziato Amendola Department of Orthopaedic Surgery, University of lowa Health Care, 200 Hawkins Drive, 0I0I8JPP, lowa City, IA 52242, USA

Email ned-amendola@uiowa.edu 
examination of these types of lesions, ${ }^{7}$ corroborating the clinical examination of the patient, and it is ideal for assessing associated lesions. A sensitivity of $78 \%$ and a specificity of $100 \%$ are reported for ACL tear radiologic diagnosis by a 1.5 Tesla MRI. ${ }^{8}$ Several ancillary findings are frequently associated with ligamentous injuries of the knee, such as osteochondral defects, occult cortical fractures, and bone bruises (BBs) or contusions. ${ }^{9}$ New sequences are currently developing in order to evaluate cartilage changes as well, such as T1 $\rho$ sequences. ${ }^{10,11}$ BBs are usually defined as an alteration of the signal intensity of the bone marrow, seen at MRI using T1-weighted and T2-weighted images, but they are best seen with fat suppression and short tau inversion recovery (STIR) sequences. ${ }^{12,13}$ Bruises of the bone have been also defined as bone marrow edema-like lesions, ${ }^{11}$ so that a differentiation can be made with the bone marrow lesions. ${ }^{14}$ The latter occur in osteoarthritis-affected knees because of the bone-on-bone stress concentration. The main cause of an articular BB appears to be the direct impact loading of the joint surfaces in conjunction with shear stress applied at the bone during a ligament rupture. ${ }^{15}$ Multiligamentous injuries have been reported in association with the ACL rupture, and $\mathrm{BBs}$ have been documented in different locations of the knee joint following acute injury or trauma. ${ }^{7,13,16}$ With respect to ACL lesions, $\mathrm{BB}(\mathrm{s})$ has been found in approximately $70 \%$ of knees with tears. ${ }^{11}$

Histologically, the BB has been rarely evaluated, but some evidence in the literature has reported that it is characterized by hemorrhage, edema, necrosis, and fibrosis. ${ }^{13,14}$ These features have been considered to result from microtrabecular fractures, which often occur during knee injury. ${ }^{11}$ In addition to these findings, chondrocyte death has been reported, also implying chondral defects of the overlying cartilage. ${ }^{11,16}$ Concerning this topic, the literature lacks strong evidence regarding the long-term changes in an ACL injured knee in relation to bone marrow changes. The location of the bone marrow lesions has been largely investigated; reporting a common location within the lateral compartment. ${ }^{17,18}$ In addition, the lesions feature a typical appearance, and the pattern of the lesion is strongly connected to the mechanism of injury. ${ }^{15}$ The natural history of a BB is not completely understood because follow-up studies, ${ }^{19,20}$ showing long- and short-term outcomes, reported variable healing patterns of these lesions over time, in terms of both their function and their anatomic sequelae.

In this review of the literature, evidence regarding MRI evaluation, localization and appearance of the bone injury, mechanism-related features, and clinical issues have been summarized. The aim of this review is to report the characteristics of the BB associated with ACL tears, its definition and classification, diagnostic tools used for assessment, its relevance on clinical outcomes, and its progression over time. In particular, we were interested in evidence regarding the long-term effects of the BB on the knee's osteochondral architecture and function.

\section{Methods}

For article research, the PubMed online database was used (http://www.ncbi.nlm.nih.gov/pubmed). The search was performed between July-August 2014. Combinations of keywords were: "bone bruise AND knee"; "bone bruise AND anterior cruciate ligament"; and "bone bruise AND osteo-chondral defects". We searched for any level of evidence studies concerning BB detection, clinically-associated features, and related injury mechanisms in patients with partial or complete ACL tears. We excluded studies in which knee BB was reported that was not associated with ACL lesions, studies on cadavers, or in vitro studies. No time interval for year of publication was set. Of each of the articles retrieved, the entire bibliography was screened in order to find studies that could be relevant for the review. Studies were retrieved in full text, analyzed, and tabulated. The studies included were classified into one of three categories: radiological; clinical; or mechanism-related studies. The study selection process was carried out as shown in Figure 1. A summary of the studies is reported in Table 1.

\section{Results \\ Definition and classification}

Lesions of bone marrow seen by MRI are usually described as an altered signal intensity of the area that involves

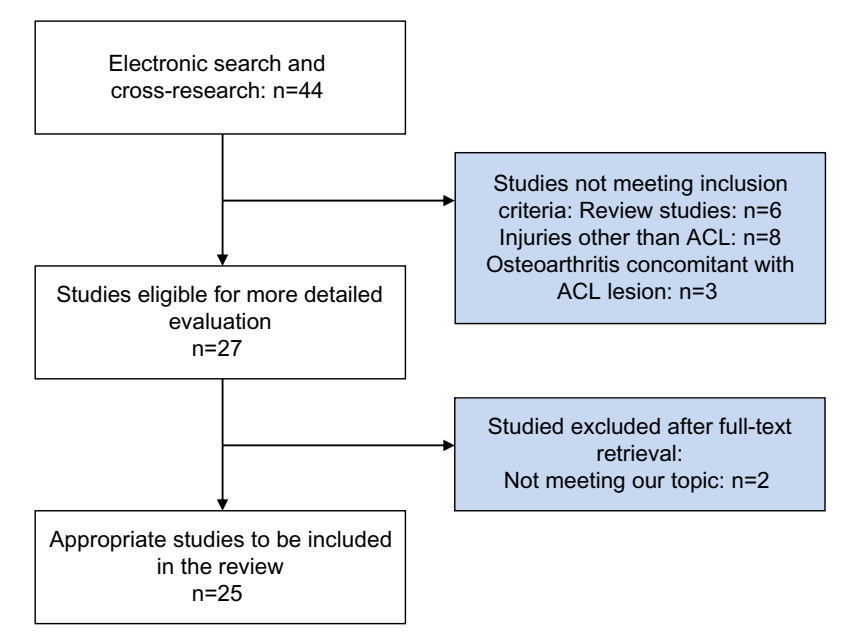

Figure I Study selection process. Abbreviations: $n$, number; $A C L$, anterior cruciate ligament. 


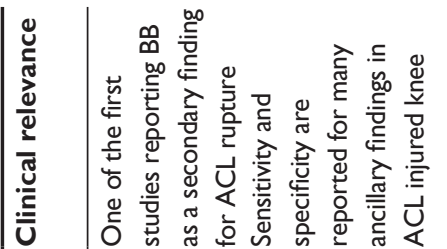
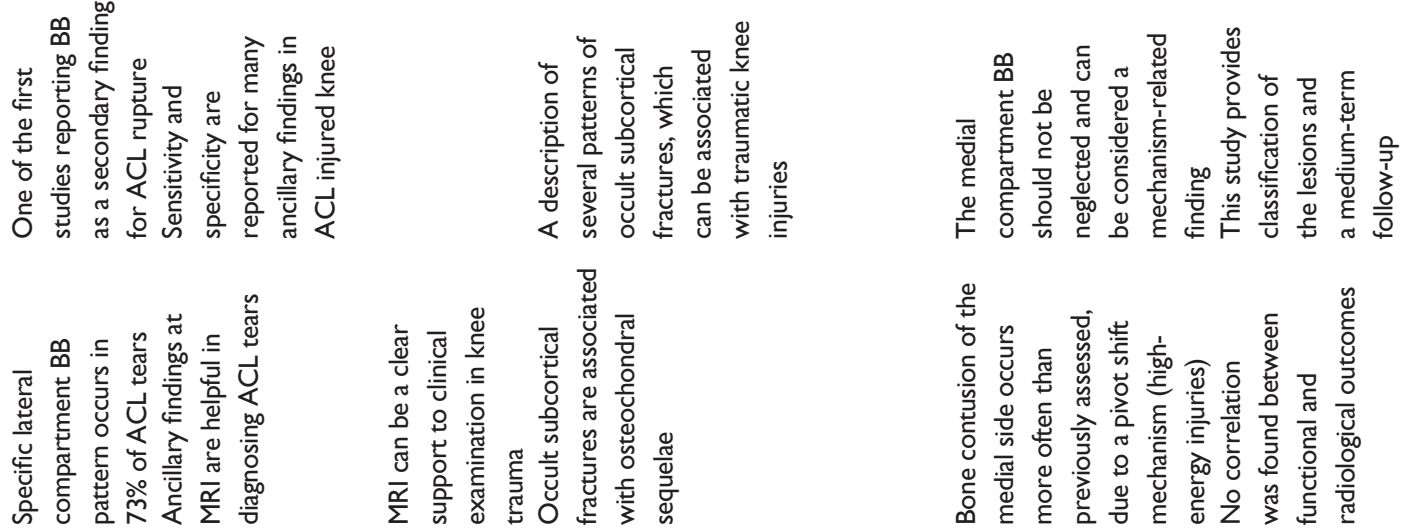

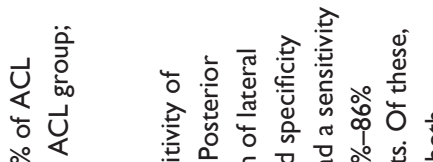

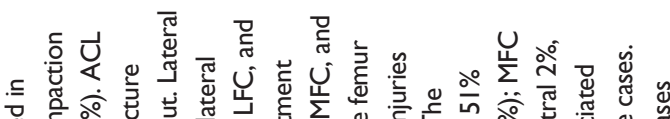

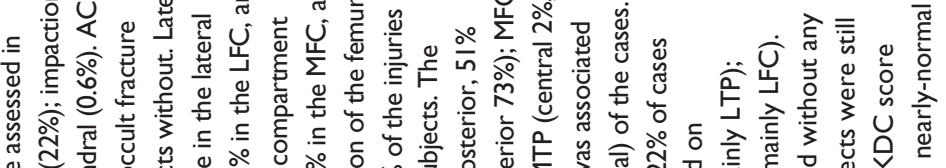

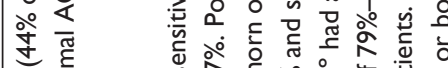

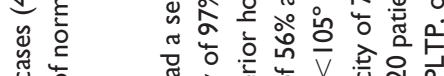

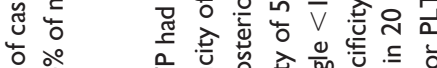

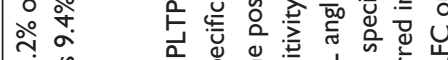

ฟั)

들

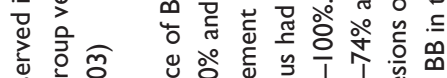

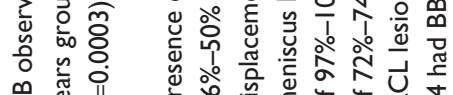

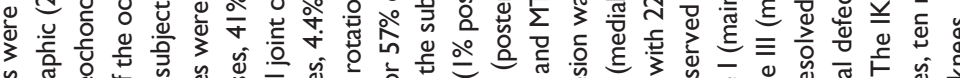

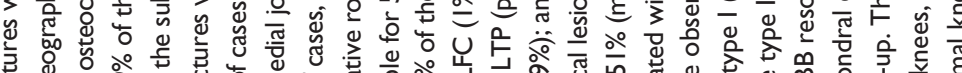

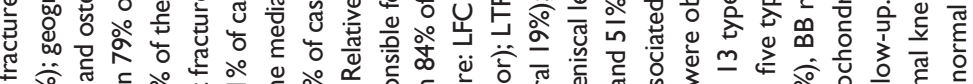

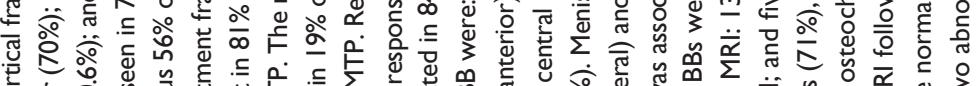

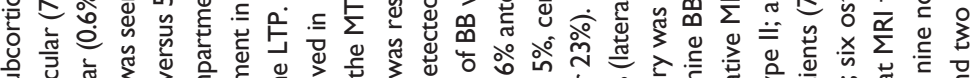

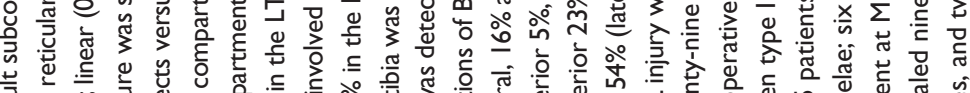

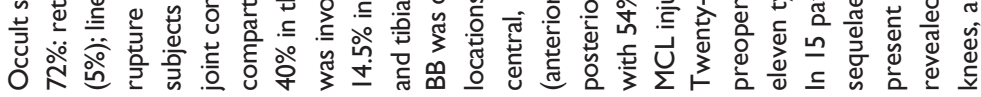

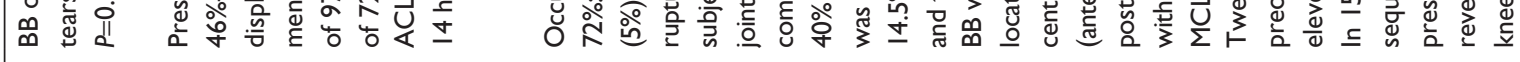

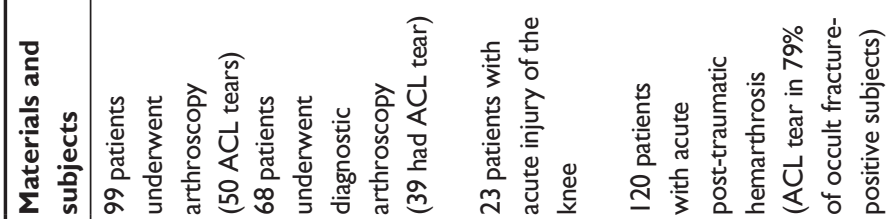

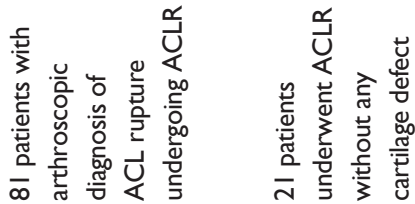

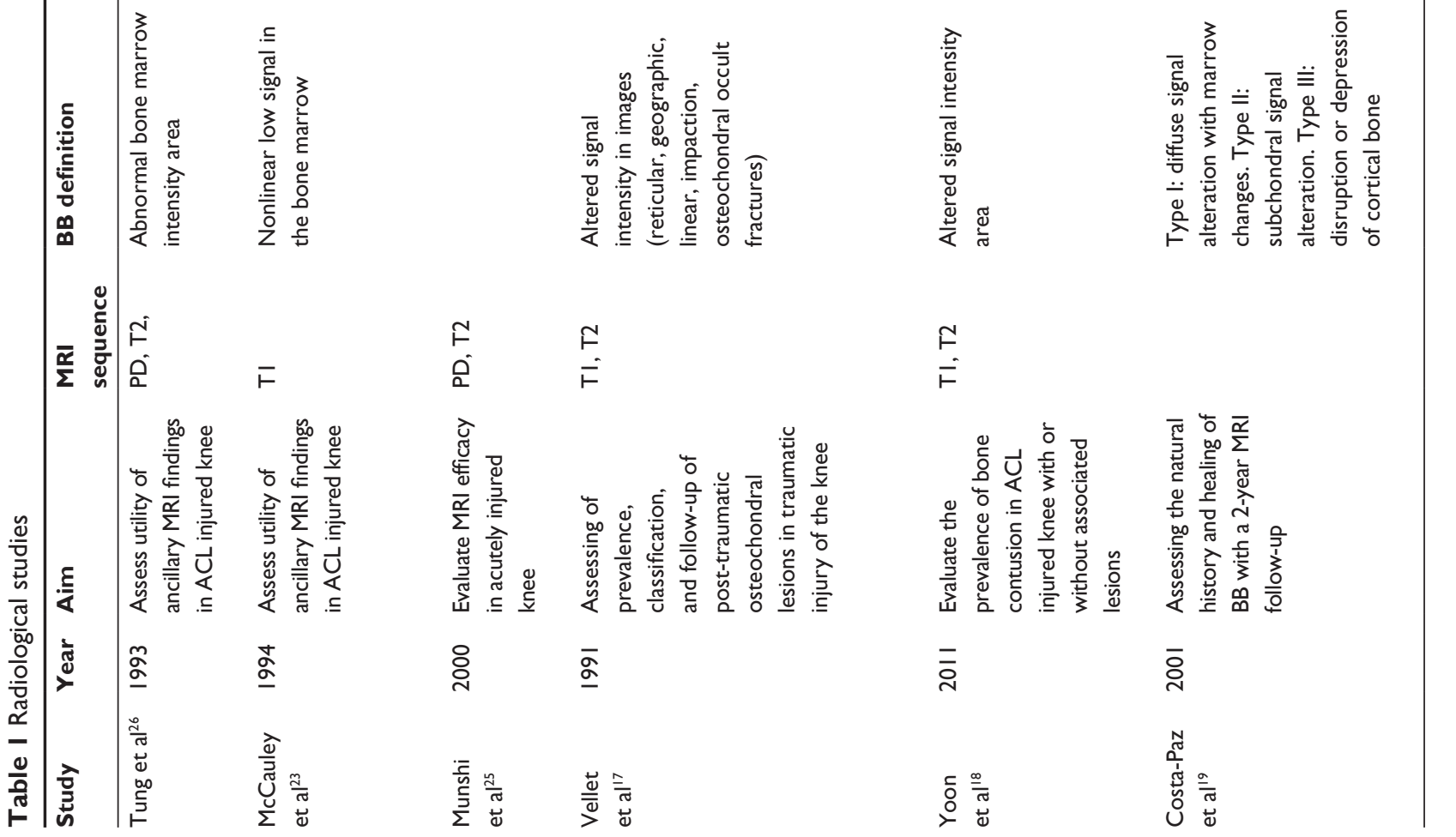




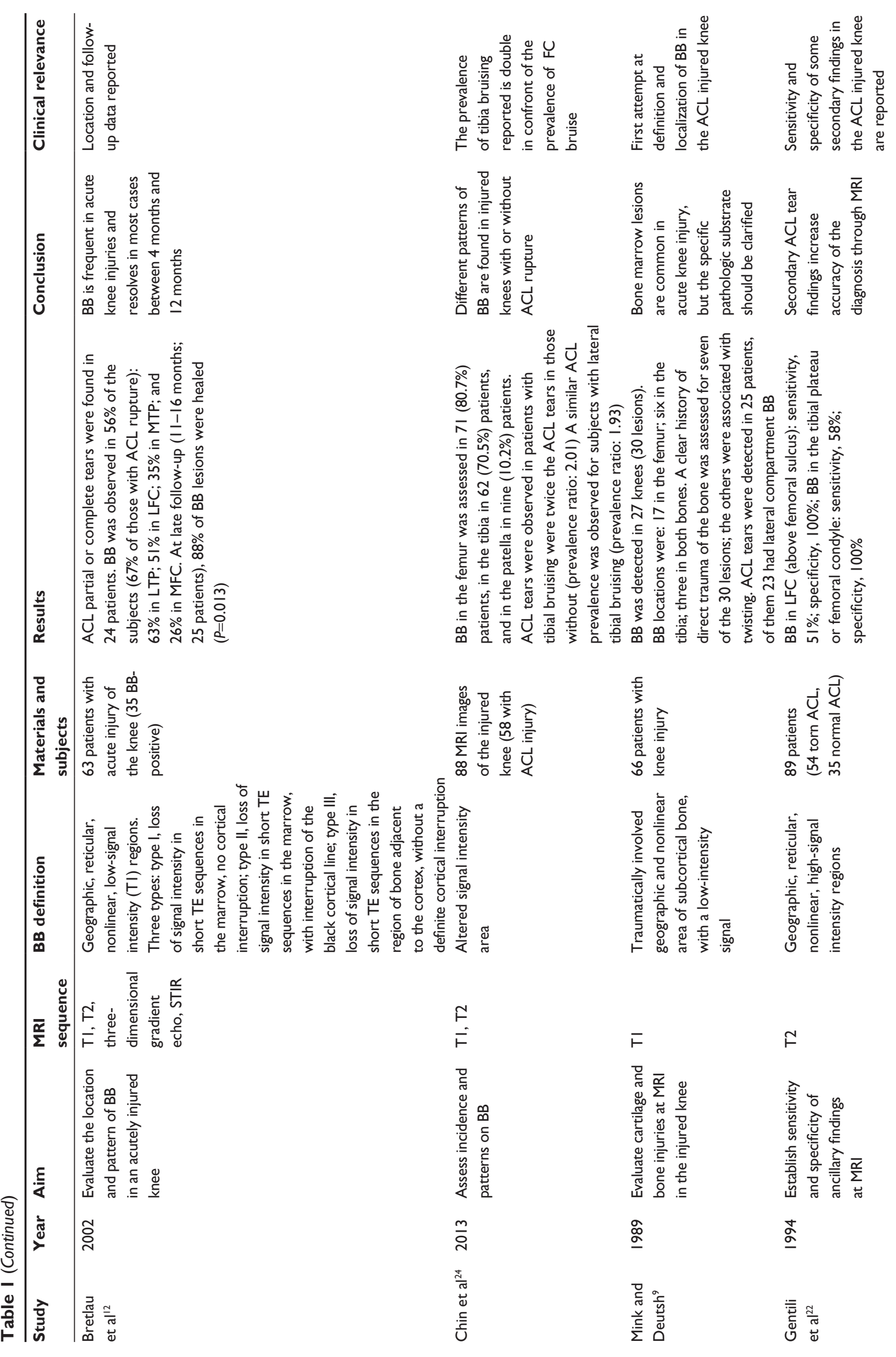




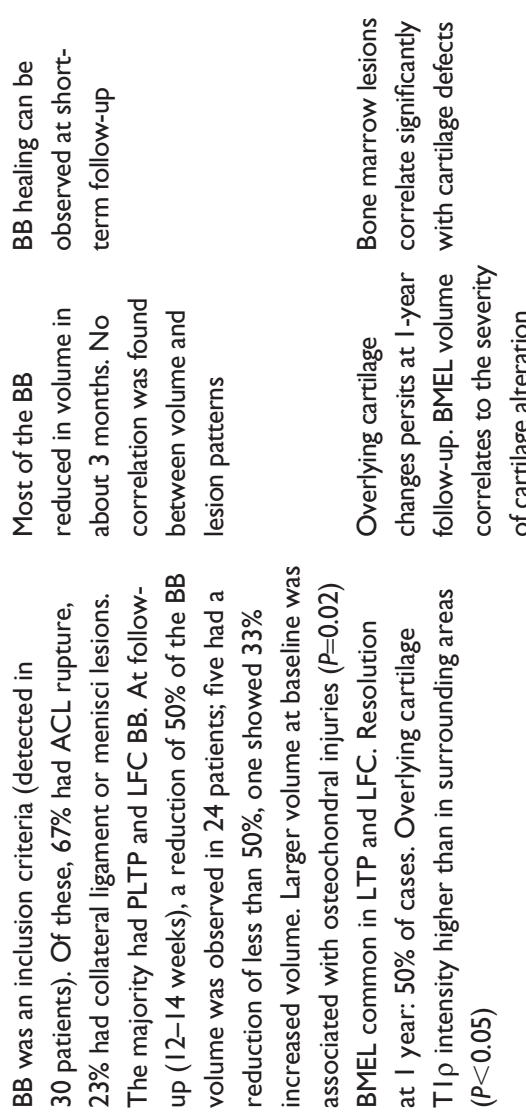

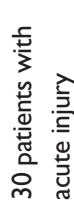

$\frac{0}{0.0}$

$\stackrel{\cong}{\mathfrak{E}}$

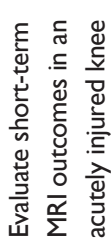

索

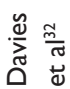
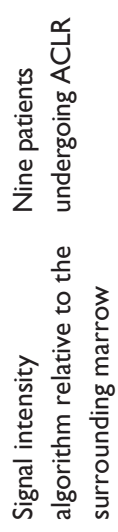

$\frac{a}{\stackrel{十}{\sim}}$

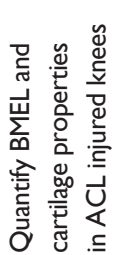

$\overline{\bar{c}}$

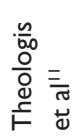

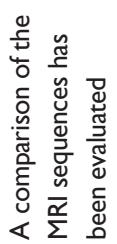

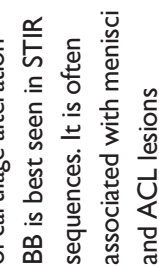

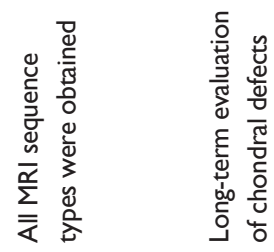

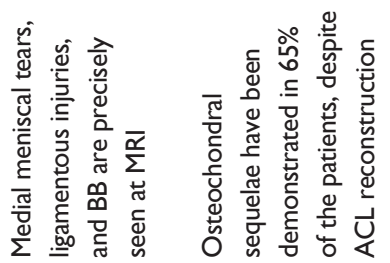

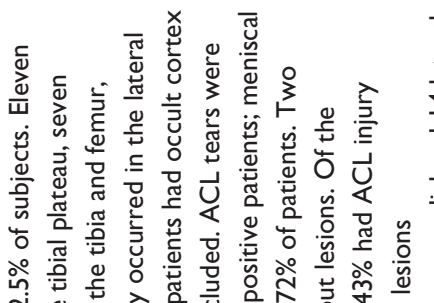

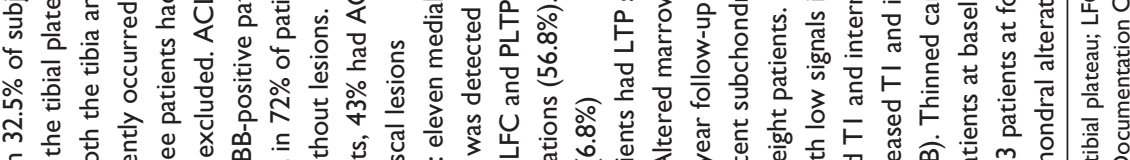

.

兽

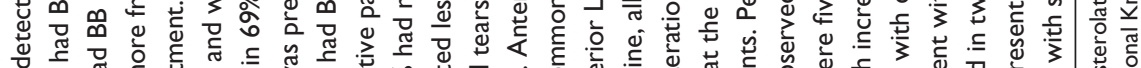

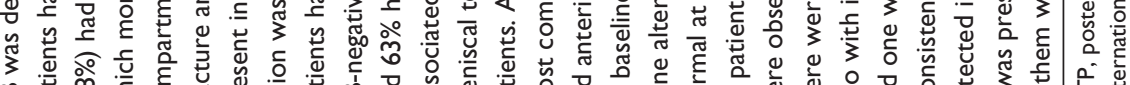

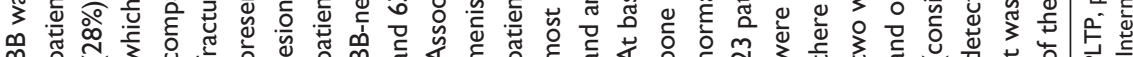

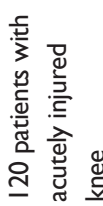

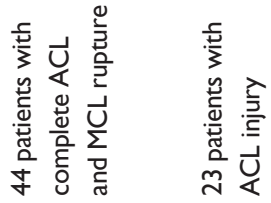

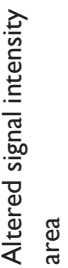

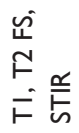

ז

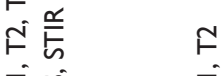

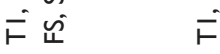

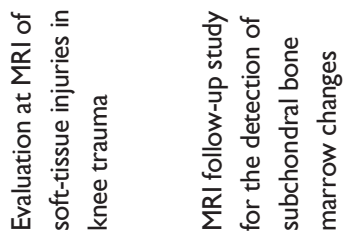

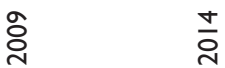

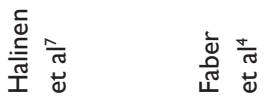

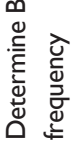

$\overline{\bar{i}}$

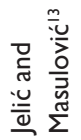




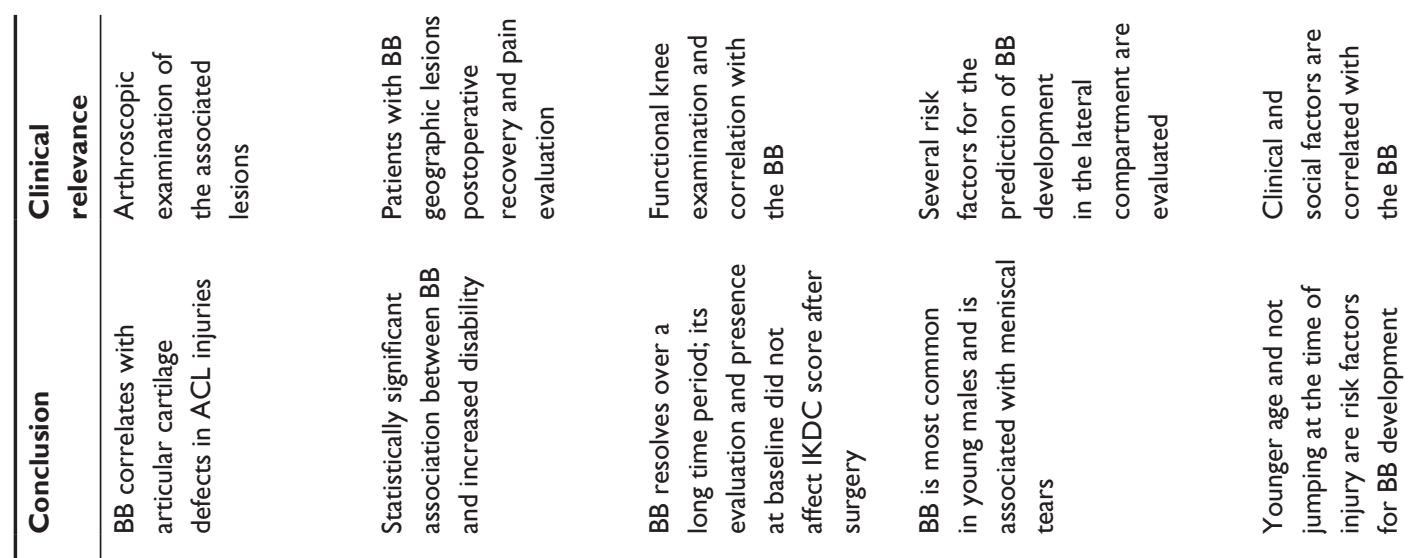

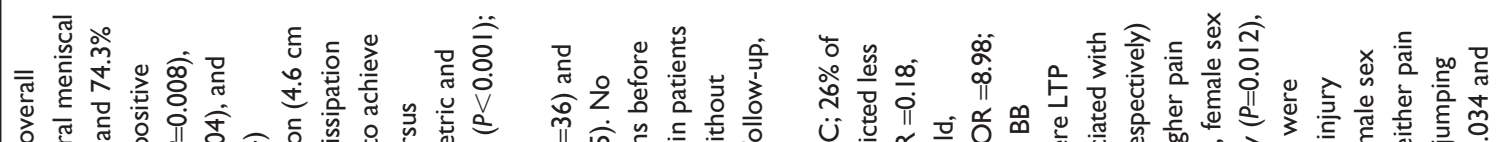

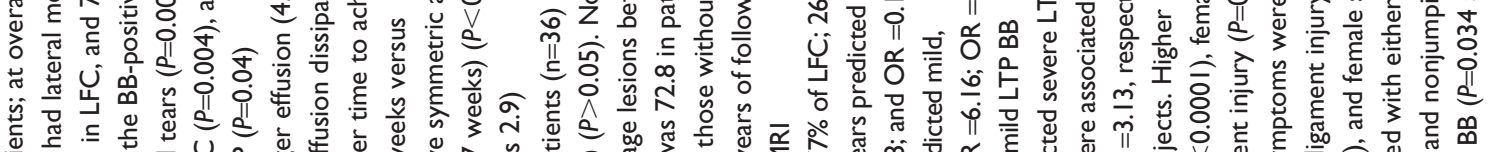

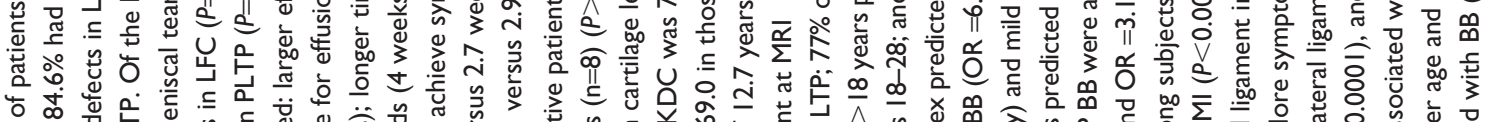

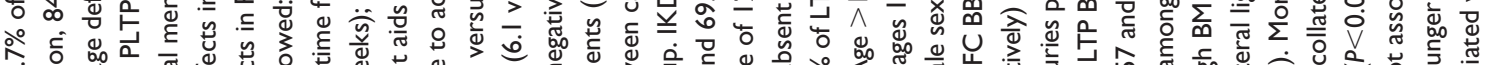

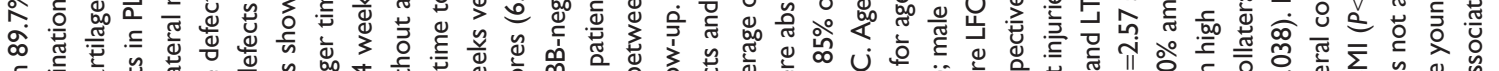

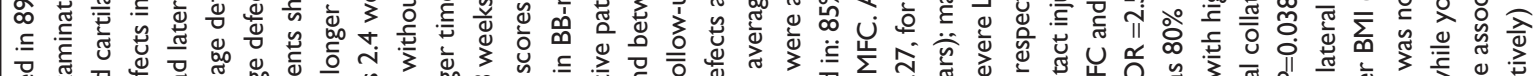

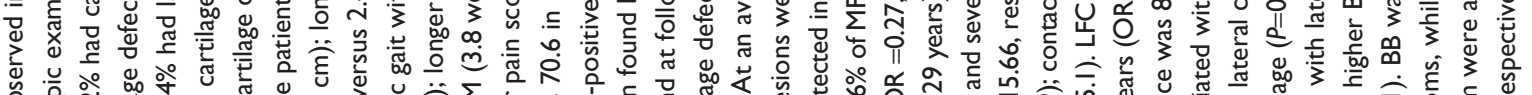

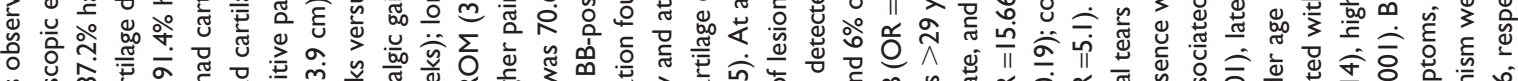

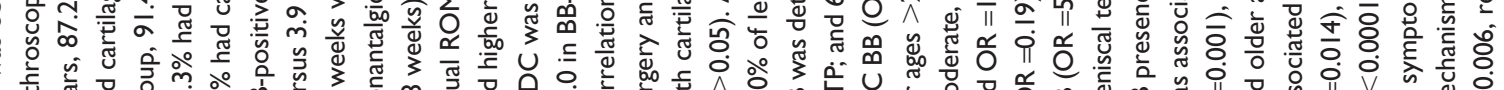

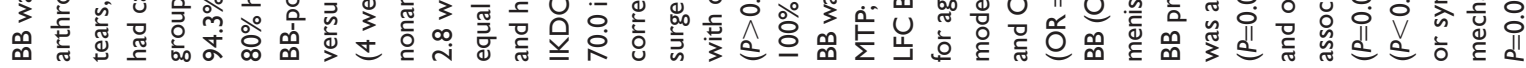

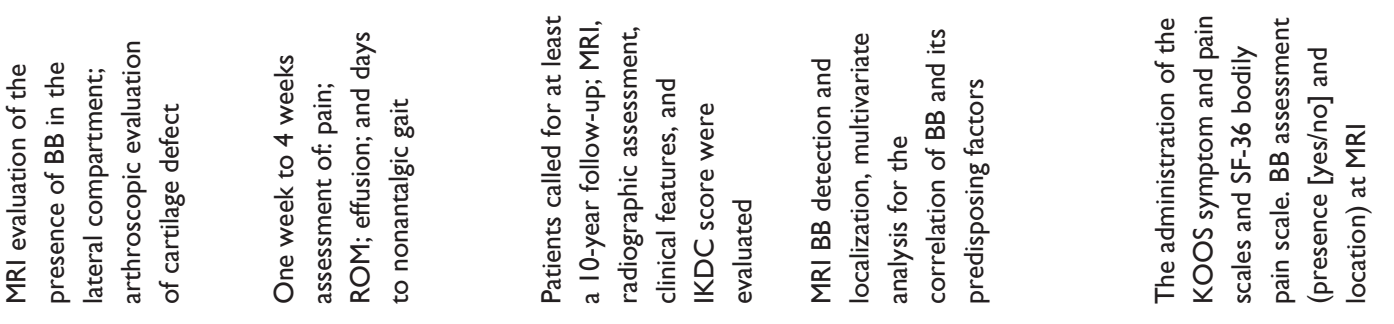
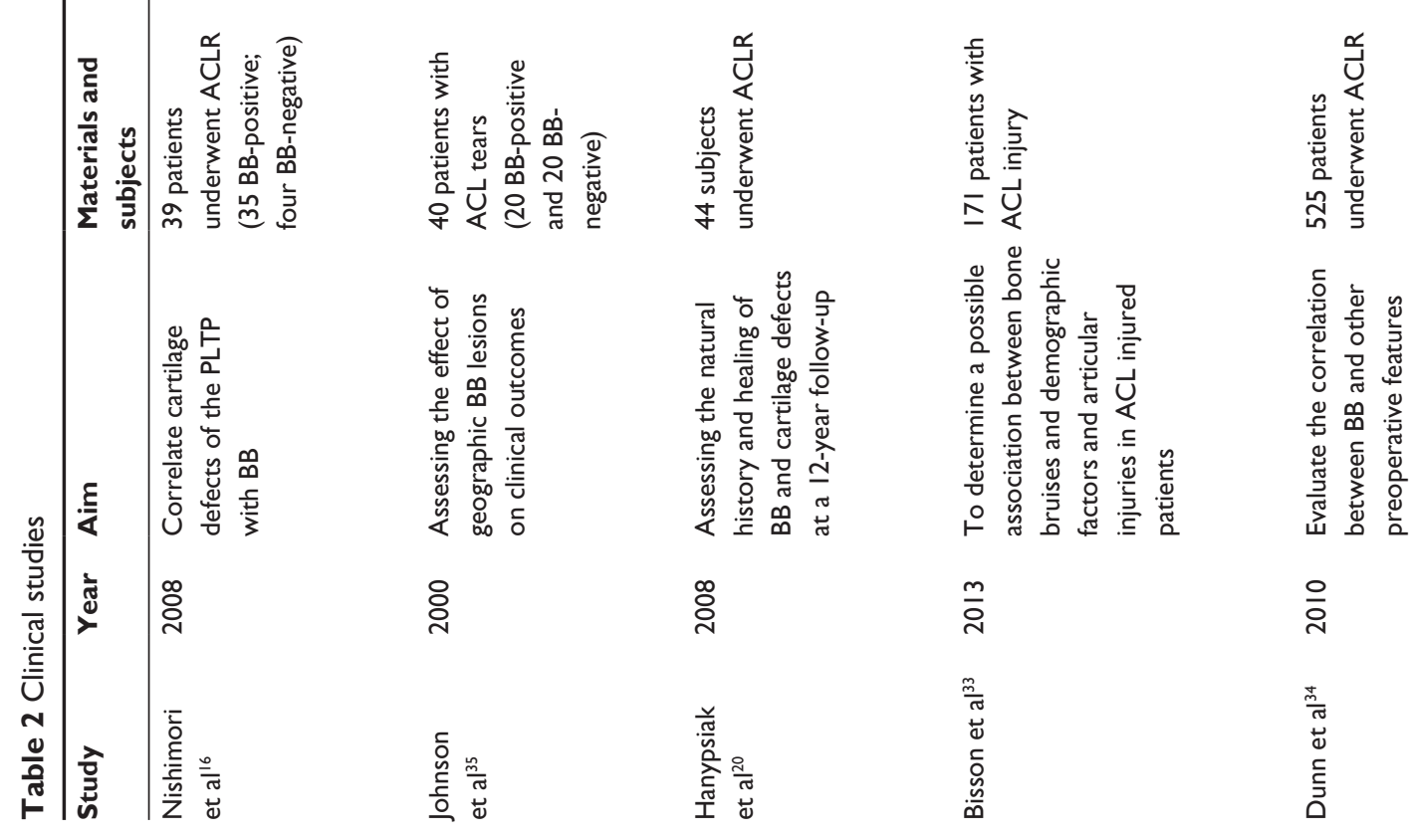


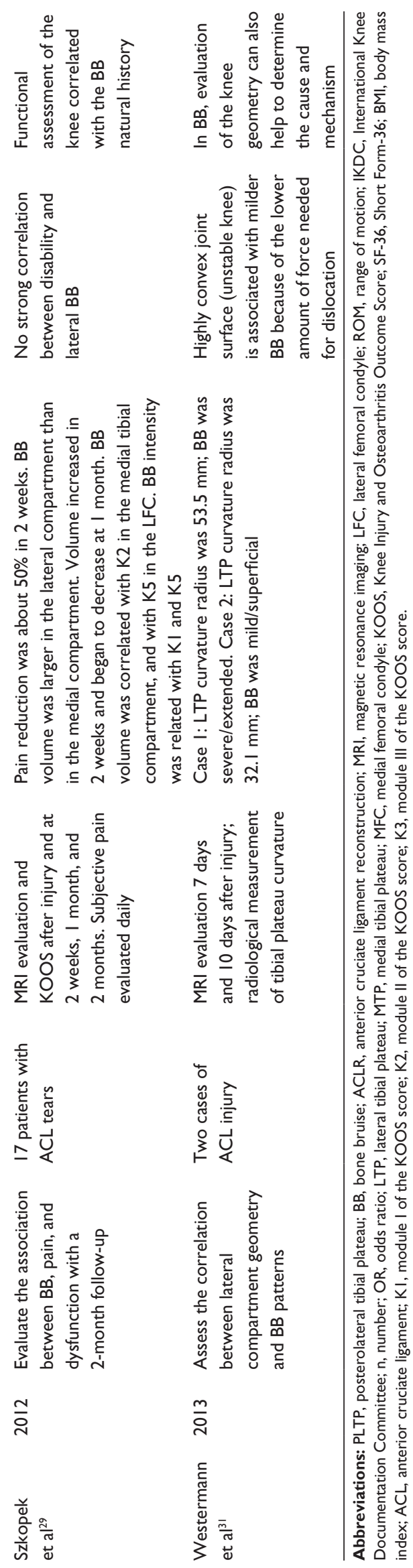

the contusion. In 1989 Mink and Deutsch ${ }^{9}$ first defined a BB as a "traumatically involved, geographic and nonlinear area of signal loss involving the subcortical bone", and Lynch et $\mathrm{a}^{21}$ proposed the first categorization. According to Lynch et al, ${ }^{21}$ three types of lesions can be observed by MRI of the knee, based on their morphology, and with all exhibiting a signal loss in short-echo time sequences (T1-weighted). Type I lesions were located in the medullary space, mainly within the epiphyseal and metaphyseal portion of the bone, without any cortical involvement; type II lesions were characterized by interruption of the black subchondral line; and type III were restricted to the subcortical area, without a definite interruption of the cortex. Occult fracture classification was later proposed by Vellet et $\mathrm{al}^{17}$ in 1991 and utilized by several successive studies for BB classification. ${ }^{12,22}$ According to this classification, three types of lesion are distinguished based on morphology and localization. Reticular fractures were reticular and serpiginous occult subcortical fractures localized in the epiphyseal and metaphyseal marrow, distant from the chondral surface. ${ }^{17}$ Geographic fractures were mainly localized near the cortex; these fractures were also divided in two subcategories: geographic I, with a coalescence pattern (not peripheral); and geographic II, coalescent, crescentic, circumscribing a central marrow fat zone close to the cortical bone.${ }^{17}$ Lastly, the linear fractures were defined as linear, $2 \mathrm{~mm}$ width subcortical lesions. ${ }^{17}$ The appearance and location of BB have also been considered by Costa-Paz et $\mathrm{al}^{19}$ in 2001, who described a further classification. Type I lesions were defined as diffuse and were associated with medullar changes, often with a reticular pattern and distant from the articular surface. Type II lesions included the focal alteration of the signal, contiguous to the articular surface. Finally, type III involved the surface, with depression or disruption of the articular contour. ${ }^{19}$

\section{Magnetic resonance imaging evaluation}

The reported MRI sensitivity and specificity are, respectively, $58 \%-61 \%$ and $86 \%-93 \%$ for BB in any bone compartment, though the specificity increases to $97 \%$ for BB in the posterior lateral tibial plateau (LTP) and $100 \%$ for the lateral femoral condyle (LFC). ${ }^{22,23}$ Radiological studies (Table 1) reported several sequences for the detection of BB; most of them evaluated BB through T1-w and T2-w sequences, ${ }^{9,12,17,22,24}$ with a shared detection of increased signal intensity in $\mathrm{T} 2-\mathrm{w}$ and decreased detection in T1-w. Proton density sequences were utilized in two studies. ${ }^{25,26}$ Other sequences, such as STIR and fat saturation, are not performed for patient examination in day-to-day clinical practice, but they have been used in some 


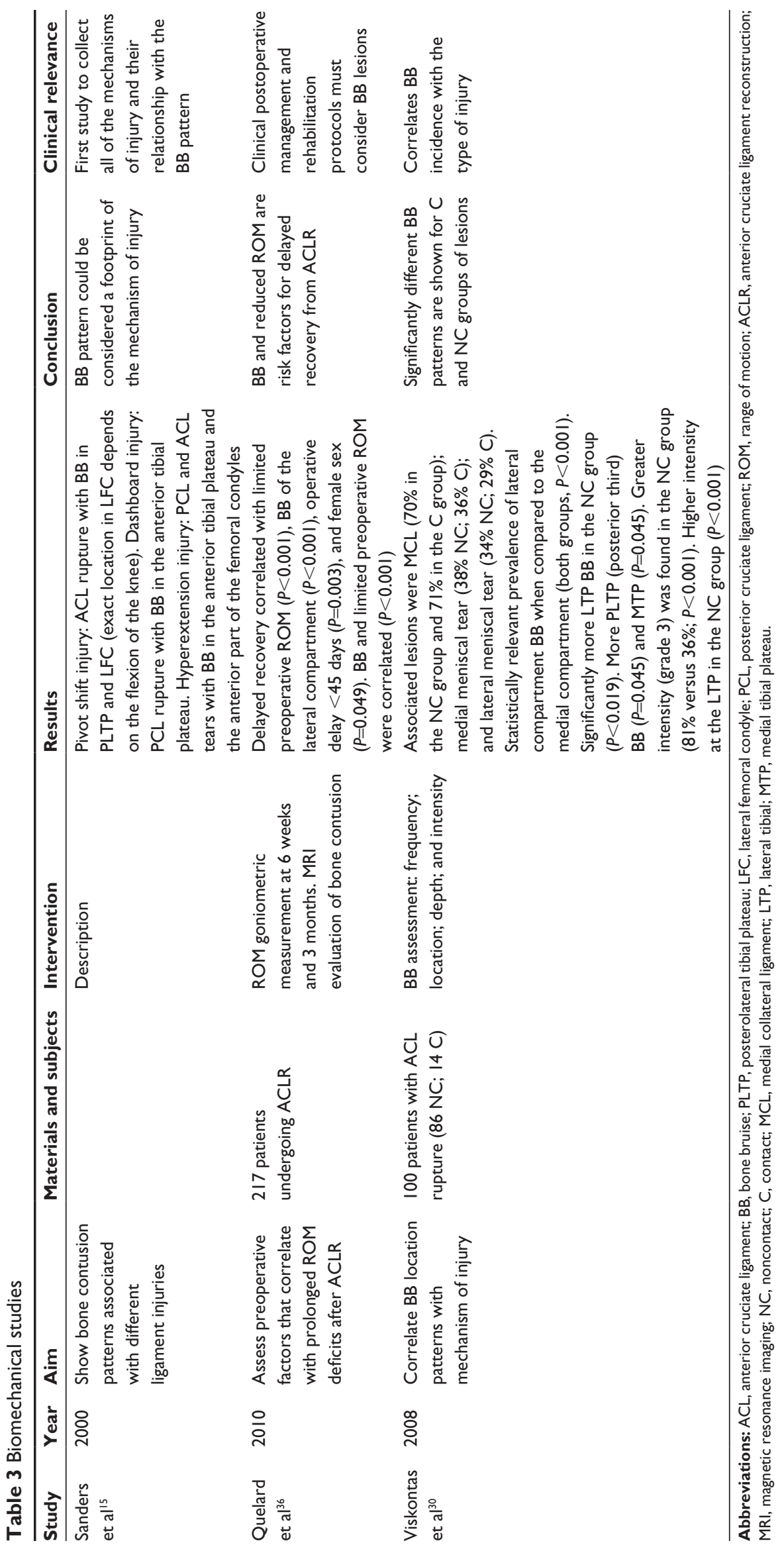


studies for the experimental examination of the knee. ${ }^{7,12,13}$ According to Jelić and Masulović, ${ }^{13}$ STIR sequences are best for detecting BB because it is able to suppress the fat bone marrow signal. Theologis et $\mathrm{a}^{11}$ utilized the $\mathrm{T} 1 \rho$ sequences for the detection of lesions in the cartilage overlying the BBinvolved area in the lateral tibia. According to these findings, an altered signal was found in the cartilage overlying the $\mathrm{BB}$ lesion when compared to the surrounding cartilage areas, at times remaining also after the $\mathrm{BB}$ had resolved. In the same study, a volume computation algorithm ${ }^{27}$ was utilized and the volume of the bruised bone can be extrapolated by setting a relative intensity threshold, which allows for the differentiation between the bruised and the normal marrow. Another method ${ }^{28}$ has been utilized by Szkopek et $\mathrm{al}^{29}$ and was based on the calculation of the diameters of the lesion (one coronal diameter and its perpendicular one) and the number of slices in which the bruise was seen.

\section{Localization}

In conjunction with ACL tears, the BB is seen in the lateral compartment of the joint - ie, the LFC and the LTP. Vellet et a ${ }^{17}$ reported $81 \%$ of $\mathrm{BB}$ in the lateral compartment $(41 \%$ LFC; 40\% LTP), while the medial compartment was involved in $19 \%$ of cases ( $4.4 \%$ in the medial femoral condyle; $14.5 \%$ in the medial tibial plateau [MTP]). Similar findings were reported by Yoon et al, ${ }^{18}$ showing an overall incidence of $84 \%$ : LFC ( $1 \%$ posterior, $51 \%$ central, $16 \%$ anterior); LTP (posterior 73\%); medial femoral condyle (anterior 5\%, central 19\%); and MTP (central 2\%, posterior 23\%). A lower incidence was reported by Bretlau et $\mathrm{al}^{12}(56 \%)$ and Jelić and Masulović $^{13}$ (32.5\%).

\section{Mechanism of injury}

Localization of the BB is strongly connected to the mechanism of injury, ${ }^{15,30}$ and many feel that the BB localization is a footprint of the mechanism itself. ${ }^{15}$ Sanders et a $1{ }^{15}$ reported a detailed analysis about the injury mechanism and BB localization. Pivot shift injuries are mainly characterized by bone contusion in the LTP and the midportion of the LFC, with a more anterior or posterior LFC localization depending on the knee flexion at the moment of the injury. ${ }^{15}$ Injury caused by hyperextension of the knee and resulting in ACL and/or posterior cruciate ligament tears is usually associated with a "kissing lesion" BB pattern, with BB localization observed in the anterior aspect of the femoral condyles and the anterior aspect of the tibial plateau, which collide against one another during hyperextension. ${ }^{15}$ It can be located medially if a varus force occurs concomitantly with the extension. ${ }^{15}$ Returning to pivot shift injuries, Westermann et $\mathrm{al}^{31}$ reported that the tibial plateau geometry affects the mechanism of injury and thus the BB pattern. In this study, the results showed that a knee with a highly convex plateau (a more unstable knee) requires less energy to dislocate; thus, bone contusion is less likely to be severe. On the other hand, a more stable knee, with a lower plateau curvature ray, needs much more energy to result in an ACL rupture, so in the event of a tear, more vigorous impact has occurred, resulting in a more severe BB. In addition to these results, it has been also reported ${ }^{30}$ that noncontact (NC) injuries result in higher bruise incidence rates when compared with the contact (C) injuries. Significantly more LTP and lateral posterior LTP BB was shown in the NC group $(P<0.019$ and $P=0.045$, respectively), and similarly in $\operatorname{MTP}(P=0.045)$. A higher intensity of bruises was found in the NC group ( $81 \%$ versus $36 \% ; P<0.001)$.

\section{Associated lesions}

The lesions, which are associated with ACL rupture in conjunction with a $\mathrm{BB}$, are meniscal tears and medial collateral ligament sprains. Nishimori et $\mathrm{al}^{16}$ reported an overall BB incidence of $89.7 \%$ of patients with acute ACL injuries. At the arthroscopic examination of these BB-positive patients, 91.4\% had lateral meniscal tears $(P=0.008), 94.3 \%$ had cartilage defects in the LFC $(P=0.004)$, and $80 \%$ had cartilage defects in the posterior LTP $(P=0.04)$. Davies et al ${ }^{32}$ reported that $67 \%$ of ACL ruptures occurred in BB-positive patients, with $23 \%$ exhibiting associated menisci or collateral ligament injuries, while Jelić and Masulović ${ }^{13}$ reported a higher incidence of meniscal tears ( $72 \%$ of the BB-positive subjects). Halinen et $\mathrm{al}^{7}$ reported eleven medial and 14 lateral meniscal tears in a cohort of 44 patients (BB prevalence: $88.6 \%$ ). Faber et al ${ }^{4}$ evaluated joint cartilage changes above marrow lesions. At baseline, all patients had LTP subchondral bone alterations at MRI, and eight had LFC marrow defects. Altered LTP marrow signal became normal at the 6.5-year follow-up in 15 of 23 patients. Persistent subchondral lesions were observed in eight patients. Of these, there were five with low signals in $\mathrm{T} 1$ and $\mathrm{T} 2$, two with increased $\mathrm{T} 1$ and intermediate $\mathrm{T} 1$, and one with decreased $\mathrm{T} 1$ and increased 2 (consistent with BB). Thinned cartilage was detected in only two patients at baseline (LFC), and it was present in 13 patients at follow-up (eight of them with subchondral bone alteration).

\section{Risk factors}

Preinjury risk factors have been not extensively investigated. According to Bisson et al, ${ }^{33}$ age $>18$ years predicted less 
LFC BB (odds ratio [OR] $=0.27$ for ages $18-28$ years; and $\mathrm{OR}=0.18$ for ages $>29$ years); male sex predicted mild, moderate, and severe LFC BB (OR =6.16; $\mathrm{OR}=8.98$; and $\mathrm{OR}=15.66$, respectively), and mild LTP BB (OR =0.19). Injury features were also analyzed, showing that $\mathrm{C}$ injuries predicted severe LTP BB $(\mathrm{OR}=5.1)$. Dunn et $\mathrm{al}^{34}$ shared the hypothesis that younger age was a risk factor $(P=0.034)$, and the authors also reported that the nonjumping mechanism of injury is mainly associated with $\mathrm{BB}(P=0.006)$.

\section{Clinical outcomes}

Two studies ${ }^{19,20}$ evaluated patients with $\mathrm{BB}$ associated with ACL rupture, utilizing the International Knee Documentation Committee (IKDC) score. Costa-Paz et al ${ }^{19}$ reported nine normal knees, ten nearly-normal knees, and two abnormal knees at a mean follow-up of 2 years among 21 patients with an original MRI that was positive for BB and ACL injury. The same study reported BB healing in $71 \%$ of patients, with cartilage defects still present in six patients. According to Hanypsiak et al, ${ }^{20}$ the mean IKDC score was 70 in 36 patients with $\mathrm{BB}$, and 70.6 in eight patients without $\mathrm{BB}(P>0.05)$. At an average follow-up of 12.7 years, all the lesions were healed at MRI examination. A similar conclusion was made by Bretlau et al, ${ }^{12}$ who conducted a follow-up between 4 months and 16 months among 64 subjects (35 were BB-positive). A clinical comparison between patients with (number $[n]=20$ ) and without $(n=20)$ BB was carried out by Johnson et al, ${ }^{35}$ indicating that patients with geographic lateral BB showed larger effusion (4.6 cm versus $3.9 \mathrm{~cm}$ ), longer time for effusion dissipation (4 weeks versus 2.4 weeks), longer time to achieve nonantalgic gait without aids (4 weeks versus 2.8 weeks), longer time to achieve symmetric and equal range of motion (ROM) (3.8 weeks versus 2.7 weeks) $(P<0.001)$, and higher pain scores (6.1 versus 2.9) when compared to subjects without $\mathrm{BB}$, respectively. ROM has also been investigated by Quelard et al, ${ }^{36}$ who assessed recovery time length in BB-positive patients. Delayed recovery correlated with limited preoperative $\operatorname{ROM}(P<0.001)$, operative delay $<45$ days $(P=0.003)$, and female sex $(P=0.049)$. BB was observed more frequently in patients with limited preoperative $\operatorname{ROM}(P<0.001)$. Szkopek et $\mathrm{al}^{29}$ assessed pain reduction (using a numeric rating scale and the K2 essay of the Knee Injury and Osteoarthritis Outcome Score [KOOS]) of about $50 \%$ in an observational follow-up of 2 weeks among patients with acute ACL rupture associated with BB. Furthermore, this study correlated the BB with the KOOS score; BB volume was correlated with $\mathrm{K} 2$ (pain) in the medial tibial compartment, and K5 (psychological and social aspects) was correlated with pain in the LFC.
BB intensity was related with K1 (swelling and mechanical symptoms) and K5.

\section{Discussion}

$\mathrm{BB}$ is associated with approximately $70 \%$ of ACL tears and can be detected with different patterns of localization and severity, consistent with the mechanism of injury. ${ }^{15}$ The BB was first described and classified by Mink and Deutsch ${ }^{9}$ in 1989 as an ancillary finding on an MRI of the injured knee. $\mathrm{BB}$ consists of bone marrow signal intensity alteration, which can be seen as a lower intensity in T1-weighted sequences, and it exhibits increased intensity in T2-weighted images. The explanation for these findings is related to the histological alterations that the bone contusion leads to. Hemorrhage and edema increase the water concentration in the involved area, which is responsible for the signal increase or decrease. STIR sequences were used in the study by Jelić and Masulović, ${ }^{13}$ who stated that these sequences are the best imaging tool for BB evaluation. From a radiological perspective, bone marrow signal is usually as intense as the signal from subcutaneous fat; therefore, when this signal is abolished, the $\mathrm{BB}$ is enhanced. The clinical issue regarding this topic is that STIR sequences are not routinely obtained for patients with knee injuries. Since BB is also easily seen using a fatsaturated T2-weighted fast spin echo exam, ${ }^{17}$ we can state that a T2-weighted STIR sequence can be used in instances where fat saturation is challenging, such as when metal is present. In addition, it may be helpful to detect and assess associated injuries at the time an ACL injury is suspected, so as to confirm the complete injury pattern. As previously reported, the mechanism of injury is directly correlated with BB location. ${ }^{15} \mathrm{NC}$ injuries seem more likely to lead to the consistent location of $\mathrm{BBs},{ }^{30}$ and knee geometry may also affect $\mathrm{BB}$ severity. ${ }^{31}$ In the literature, it is also reported that the medial side $\mathrm{BB}$ should raise concerns for a possible multiligamentous injury. ${ }^{37}$

From an overall perspective, the BB pattern can be evaluated as a result of the specific bone contusion mechanism that was in place during the injury; this can lead to a better clinical examination of the patients in order to retrieve lesions that are specifically associated with that injury type. Clinically, higher pain scores were observed in patients with BBs when compared with the scores of those without, ${ }^{35}$ but functionally, the BB in ACL injured knees does not correlate with IKDC scores. ${ }^{19,20}$ Based on this review of the literature, at medium- and longterm follow-up, there does not appear to be any relationship between BB pattern and clinical knee function. However, Theologis et $\mathrm{al}^{11}$ reported that defects of the overlying joint 
cartilage can still be seen at a 12-month follow-up, despite healing of the bruise. In addition to this, Faber et $\mathrm{al}^{4}$ reported a further development of cartilage lesions following subchondral marrow changes. Therefore, longer-term follow-up is necessary to clearly outline the natural history of the BB and the overlying cartilage. There appears to be some interest in assessing BB volume and size,,$^{11,27,29}$ and this may be associated with chondral defects and long-term functional knee scores. At this point, volume analysis is not of any routine clinical relevance, but it may be related to overall joint injury and eventual long-term impact on joint function. Simple algorithm-based software ${ }^{28}$ could be used for the follow-up of these lesions in order to monitor their reduction over time, as well as to clear the patient to return to full activity levels. An experimental algorithm was also developed, ${ }^{11,27}$ but its complexity limits its use to scientific research only.

With respect to chondral defects, Theologis et $\mathrm{al}^{11}$ reported that microscopic lesions of the overlying joint cartilage can be still seen after BB healing. Clinical studies concerning this topic should be carried out, in order to correlate morphological changes in the knee architecture (osseous and cartilage structures) and the patient's long-term functional outcomes. At this point, there appears to be a lack of evidence as to whether the BB should be regarded as a lesion that needs treatment or not, especially in terms of the possible effects of microtrabecular fractures on the biomechanics of knee joint homeostasis. Regrettably, the reviewed studies were really different in terms of the sequences used to assess the BB, the cohort size, and the outcomes evaluated; therefore, we were not able to compare results. Furthermore, most of the studies were of a low level of evidence, and this affected the quality of the findings. In addition to these limitations, the unsystematic character of this review may have led to some relevant papers being missed.

\section{Conclusion}

In conclusion, although there seems to be quite a few studies on various aspects of BBs, many questions remain, especially in terms of the effect of the BB on long-term joint function, its relationship to outcomes following ACL injury, and the development of post-traumatic osteoarthritis. We can state that currently, BB of the knee is an ancillary radiographic finding of the ACL and other knee multiligamentous injuries. It is often associated with other joint injuries, in addition to the ACL, as a conglomeration of injury severity. Therefore, the impact of the BB on global joint injury (rather than in isolation) may be of greater significance. Although its presence yields higher pain levels, no correlations have been solidly made with functional outcomes. Most follow-up studies of BBs are short term when compared to the length of time it takes to develop post-traumatic osteoarthritis, so it still remains unclear if the initial joint injury and $\mathrm{BB}$ have a direct relationship to long-term function. Post-traumatic osteoarthritis of the knee is a common sequelae of ACL injury, and identifying predisposing factors early in the course of the disease is essential as a means of developing and instituting preventative measures.

\section{Disclosure}

The authors report no conflicts of interest in this work.

\section{References}

1. Nicolini AP, de Carvalho RT, Matsuda MM, Sayum J, Cohen M. Common injuries in athletes' knee: experience of a specialized center. Acta Ortop Bras. 2014;22(3):127-131.

2. Sutton KM, Bullock JM. Anterior cruciate ligament rupture: differences between males and females. J Am Acad Orthop Surg. 2013;21(1): 41-50.

3. Barenius B, Ponzer S, Shalabi A, Bujak R, Norlén L, Eriksson K. Increased risk of osteoarthritis after anterior cruciate ligament reconstruction: a 14-year follow-up study of a randomized controlled trial. Am J Sports Med. 2014;42(5):1049-1057.

4. Faber KJ, Dill JR, Amendola A, Thain L, Spouge A, Fowler PJ. Occult osteochondral lesions after anterior cruciate ligament rupture. Six-year magnetic resonance imaging follow-up study. Am J Sports Med. 2014; 27(4):489-494.

5. Lohmander LS, Englund PM, Dahl LL, Roos EM. The long-term consequence of anterior cruciate ligament and meniscus injuries: osteoarthritis. Am J Sports Med. 2007;35(10):1756-1769.

6. Wen C, Lohmander LS. Osteoarthritis: Does post-injury ACL reconstruction prevent future OA? Nat Rev Rheumatol. 2014;10(10):577-578.

7. Halinen J, Koivikko M, Lindahl J, Hirvensalo E. The efficacy of magnetic resonance imaging in acute multi-ligament injuries. Int Orthop. 2009;33(6):1733-1738.

8. Van Dyck P, Vanhoenacker FM, Lambrecht V, et al. Prospective comparison of 1.5 and 3.0-T MRI for evaluating the knee menisci and ACL. J Bone Joint Surg Am. 2013;95(10):916-924.

9. Mink JH, Deutsch AL. Occult cartilage and bone injuries of the knee: detection, classification, and assessment with MR imaging. Radiology. 1989;170(3 Pt 1):823-829.

10. Gupta R, Virayavanich W, Kuo D, et al. MR T1 $\rho$ quantification of cartilage focal lesions in acutely injured knees: correlation with arthroscopic evaluation. Magn Reson Imaging. Epub August 8, 2014.

11. Theologis AA, Kuo D, Cheng J, et al. Evaluation of bone bruises and associated cartilage in anterior cruciate ligament-injured and -reconstructed knees using quantitative $t(1 \rho)$ magnetic resonance imaging: 1-year cohort study. Arthroscopy. 2011;27(1):65-76.

12. Bretlau T, Tuxøe J, Larsen L, Jørgensen U, Thomsen HS, Lausten GS. Bone bruise in the acutely injured knee. Knee Surg Sports Traumatol Arthrosc. 2002;10(2):96-101.

13. Jelić D, Masulović D. Bone bruise of the knee associated with the lesions of anterior cruciate ligament and menisci on magnetic resonance imaging. Vojnosanit Pregl. 2011;68(9):762-766.

14. Hernández-Molina G, Guermazi A, Niu J, et al. Central bone marrow lesions in symptomatic knee osteoarthritis and their relationship to anterior cruciate ligament tears and cartilage loss. Arthritis Rheum. 2008;58(1):130-136.

15. Sanders TG, Medynski MA, Feller JF, Lawhorn KW. Bone contusion patterns of the knee at MR imaging: footprint of the mechanism of injury. Radiographics. 2000;20 Spec No:S135-S151. 
16. Nishimori M, Deie M, Adachi N, et al. Articular cartilage injury of the posterior lateral tibial plateau associated with acute anterior cruciate ligament injury. Knee Surg Sports Traumatol Arthrosc. 2008;16(3):270-274.

17. Vellet AD, Marks PH, Fowler PJ, Munro TG. Occult posttraumatic osteochondral lesions of the knee: prevalence, classification, and shortterm sequelae evaluated with MR imaging. Radiology. 1991;178(1): 271-276.

18. Yoon KH, Yoo JH, Kim KI. Bone contusion and associated meniscal and medial collateral ligament injury in patients with anterior cruciate ligament rupture. J Bone Joint Surg Am. 2011;93(16):1510-1518.

19. Costa-Paz M, Muscolo DL, Ayerza M, Makino A, Aponte-Tinao L. Magnetic resonance imaging follow-up study of bone bruises associated with anterior cruciate ligament ruptures. Arthroscopy. 2001;17(5): 445-449.

20. Hanypsiak BT, Spindler KP, Rothrock CR, et al. Twelve-year follow-up on anterior cruciate ligament reconstruction: long-term outcomes of prospectively studied osseous and articular injuries. Am J Sports Med. 2008;36(4):671-677.

21. Lynch TC, Crues JV 3rd, Morgan FW, Sheehan We, Harter LP, Ryu R. Bone abnormalities of the knee: prevalence and significance at MR imaging. Radiology. 1989;171(3):761-766.

22. Gentili A, Seeger LL, Yao L, Do HM. Anterior cruciate ligament tear: indirect signs at MR imaging. Radiology. 1994;193(3):835-840.

23. McCauley TR, Moses M, Kier R, Lynch JK, Barton JW, Jokl P. MR diagnosis of tears of anterior cruciate ligament of the knee: importance of ancillary findings. AJR Am J Roentgenol. 1994;162(1):115-119.

24. Chin YC, Wijaya R, Chong LR, Chang HC, Lee YH. Bone bruise patterns in knee injuries: where are they found? Eur J Orthop Surg Traumatol. Epub September 22, 2013.

25. Munshi M, Davidson M, MacDonald PB, Froese W, Sutherland K. The efficacy of magnetic resonance imaging in acute knee injuries. Clin $J$ Sport Med. 2000;10(1):34-39.

26. Tung GA, Davis LM, Wiggins ME, Fadale PD. Tears of the anterior cruciate ligament: primary and secondary signs at MR imaging. Radiology. 1993;188(3):661-667.

27. Li X, Ma BC, Bolbos RI, et al. Quantitative assessment of bone marrow edema-like lesion and overlying cartilage in knees with osteoarthritis and anterior cruciate ligament tear using MR imaging and spectroscopic imaging at 3 Tesla. J Magn Reson Imaging. 2008;28(2):453-461.
28. Huttner HB, Steiner T, Hartmann M, et al. Comparison of ABC/2 estimation technique to computer-assisted planimetric analysis in warfarin-related intracerebral parenchymal hemorrhage. Stroke. 2006;37(2):404-408.

29. Szkopek K, Warming T, Neergaard K, Jørgensen HL, Christensen HE, Krogsgaard M. Pain and knee function in relation to degree of bone bruise after acute anterior cruciate ligament rupture. Scand J Med Sci Sports. 2012;22(5):635-642.

30. Viskontas DG, Giuffre BM, Duggal N, Graham D, Parker D, Coolican M. Bone bruises associated with ACL rupture: correlation with injury mechanism. Am J Sports Med. 2008;36(5):927-933.

31. Westermann RW, Wolf BR, Wahl CJ. Does lateral knee geometry influence bone bruise patterns after anterior cruciate ligament injury? A report of two cases. Iowa Orthop J. 2013;33:217-220.

32. Davies NH, Niall D, King LJ, Lavelle J, Healy JC. Magnetic resonance imaging of bone bruising in the acutely injured knee - short-term outcome. Clin Radiol. 2004;59(5):439-445.

33. Bisson LJ, Kluczynski MA, Hagstrom LS, Marzo JM. A prospective study of the association between bone contusion and intra-articular injuries associated with acute anterior cruciate ligament tear. Am J Sports Med. 2013;41(8):1801-1807.

34. Dunn WR, Spindler KP, Amendola A, et al; MOON ACL Investigation. Which preoperative factors, including bone bruise, are associated with knee pain/symptoms at index anterior cruciate ligament reconstruction (ACLR)? A Multicenter Orthopaedic Outcomes Network (MOON) ACLR Cohort Study. Am J Sports Med. 2010;38(9):1778-1787.

35. Johnson DL, Bealle DP, Brand JC Jr, Nyland J, Caborn DN. The effect of a geographic lateral bone bruise on knee inflammation after acute anterior cruciate ligament rupture. Am J Sports Med. 2000;28(2): $152-155$.

36. Quelard B, Sonnery-Cottet B, Zayni R, Ogassawara R, Prost T, Chambat $\mathrm{P}$. Preoperative factors correlating with prolonged range of motion deficit after anterior cruciate ligament reconstruction. Am J Sports Med. 2010;38(10):2034-2039.

37. Geeslin AG, LaPrade RF. Location of bone bruises and other osseous injuries associated with acute grade III isolated and combined posterolateral knee injuries. Am J Sports Med. 2010;38(12): 2502-2508.
Open Access Journal of Sports Medicine

\section{Publish your work in this journal}

Open Access Journal of Sports Medicine is an international, peer-reviewed, open access journal publishing original research, reports, reviews and commentaries on all areas of sports medicine. The manuscript management system is completely online and includes a very quick and fair peer-review system.

\section{Dovepress}

Visit http://www.dovepress.com/testimonials.php to read real quotes from published authors. 